[pedagogical]

Diamond Open Access

\title{
Golden Rules for Referees
}

\author{
Open Collaboration*† \\ December 30, 2019
}

\begin{abstract}
This manuscript serves as a pedagogical guideline for referees during the peer-reviewing process.
\end{abstract}

keywords: peer-review, referee, ethics

\section{Introduction}

1. Producing knowledge and helping to develop science is a holy endeavor.

2. A few numbers of referees (Hopefully!) use their ego to produce predatory comments during peer-review.

3. In the following section, we present some golden rules [1] that are expected in the peer-reviewing process [2].

*All authors with their affiliations appear at the end of this paper.

†Corresponding author: mplobo@uft.edu.br| Join the Open Collaboration 


\section{Golden Rules for a Healthier Science}

4. Be ethical.

5. Be respectful.

6. Leave your ego outside the peer-reviewing process.

7. Refuse to peer-review an article whenever it is not within your expertise.

8. Refuse to peer-review an article whenever your opinion does not agree with the strategies accomplished by the authors.

9. Avoid general and unsubstantial arguments.

10. Provide one or more examples for each statement that embodies the referee's main argument.

11. Rejecting the publication of an article because one of the referee's article was not cited in it is extremely unethical.

12. The identity of the referee should be revealed.

13. The anonymity supports abusive behavior.

\section{Final Remarks}

14. If we always remember these golden rules and use them as guidelines during the peer-review, science becomes more collaborative in a much more profound sense.

\section{Open Invitation}

Review, add content, and co-author this article. Join the Open Collaboration. Send your contribution to mplobo@uft.edu.br. 


\section{Ethical conduct of research}

This original work was pre-registered under the OSF Preprints [3], please cite it accordingly [4]. This will ensure that researches are conducted with integrity and intellectual honesty at all times and by all means.

\section{References}

[1] Glen, A. S. "A New 'Golden Rule' for Peer Review." Bulletin of the Ecological Society of America 95.4 (2014): 431-434.

[2] Liebowitz, Jay. A guide to publishing for academics: Inside the publish or perish phenomenon. Auerbach Publications, 2015.

[3] COS. Open Science Framework. https://osf.io

[4] Lobo, Matheus P. "Golden Rules for Referees." OSF Preprints, 24 Dec. 2019. https://doi.org/10.31219/osf.io/grz9j

\section{The Open Collaboration}

Matheus Pereira Lobo (lead author, mplobo@uft.edu.br) $)^{1,2}$

${ }^{1}$ Federal University of Tocantins (Brazil); ${ }^{2}$ Universidade Aberta (UAb, Portugal) 